Discussion to 126.

\title{
Trasylol on Experimental Brain Edema
}

\author{
Hideo AOKI and Tomiyasu Egami \\ 2nd Surgical Department, Yamaguchi University Medical School
}

Experimental brain edema was prepared by the expradural balloon method with rabbit. Intravenous injection of trasylol (25,000 uts) was carried out twice; the first, at the filling of the extradurally introduced balloon with saline solution and the second, at the emptying this balloon after 24 hours. Then 24 hours later from the last injection, the experimenal animals were sacrificed and the destruction of the blood brain barrier was examined with fluorescein method.

7 rabbits were used in this experiment. Of the 4 cases no fluorescein was observed, and of the 2 cases slight staining was found. Last one case was stained by dye as same grade in control. Therefore, it seems that the trasylol has the protective effect on the blood brain barrier in this type of brain edema since in about $68 \%$ cass the staining of the brain was inhibited.

Trasylol is the inhibitor for the many kinds of proteolytic enzymes. It has been accepted that this drug has three main sites of action such as kinin system, plasmin system and blood coagulation system. On the other hand, the action mechanismus of this drug on the blood brain barrier is very interest problem which should be elucidated.

\section{Central Syndrome; Rostral-Caudal Deterioration in Acute Head Injury}

Tetsuya Leslie Sasabe, Yoshikazu Iwata, Susumu Nakatani, Masaya Ohta, Mamoru Taneda, Kohei Hayashi and Ichiro Tahara

Department of Neurological Surgery, Osaka National Hospital

Broken compensation following acute supratentorial injury may show either uncal syndrome or central syndrome, with a few exceptions. It is evident for an appearance of uncal syndrome to suggest a prompt surgical intervention. On the other hand there seem a few possibilities to lose surgical interventions under stuporous or comatose state without anisocoria and focal sign due to a secondary lesion. As to central syndrome there are detailed descriptions done by Drs. Plum, Posner and McNealey. Central syndrome has been divided into early and late 